\title{
Author Index to Volume 38
}

Aarnoudse, JG, 528

Abbag, F, 55

Abu-Saad, HH, 194

Adams, S, 1009

Adamson, TM, 533

Ådén, U, 312

Agosti, V, 564

Agostini, C, 262

Aguzzi, A, 113

Akino, T, 676

Albertsson-Wikland, K, 733

Aldoretta, PA, 754

Alessandri, G, 792

Almotrefi, AA, 55

Alvarez-Novoa, R, 149

Amat, M, 81

Ambruso, DR, 993

Anderson, SM, 754

Antakly, T, 506

Aranda, JV, 981

Ascuitto, RJ, 228

Ashwal, S, 652

Baan, J, Jr., 938

Baba, M, 187

Baker, J, 133

Bakr, S, 55

Ballard, PL, 668

Bancalari, E, 704

Banovcin, P, 218

Barbadora, KA, 988

Bartelds, B, 243

Bartolomé, J, 618

Battaglia, FC, 775

Bechensteen, AG, 729

Beers, MF, 668

Beharry, K, 981

Beishuizen, A, 404

Beitins, IZ, 763

Bekesi, JG, 390

Bel, F, 243

Belgorosky, A, 592

Bellù, R, 262

Bentele, KHP, 298

Berard, DJ, 67

Berensztein, E, 592

Berger, PJ, 533

Berger, RMF, 36

Berkeland, JE, 878

Bernardini, I, 579

Bernasconi, S, 91

Berry, LM, 709

Berry-Kravis, E, 638

Berseth, CL, 133

Bitsch, I, 585

Björkholm, M, 7

Black, SM, 912

Blanch, N, 533

Blanckaert, N, 349

Bland, RD, 30

Blommaart, PJE, 1018
Boat, TF, 42

Boekkooi, PF, 938

Boesch, C, 657

Bohnsack, JF, 1003

Bona, E, 312

Boucher, RC, 42

Bouman, HGA, 342

Boyce, WT, 1009

Bravo, P, 156

Broekhuizen, MLA, 342

Broerse, HM, 808

Brönnegård, M, 418

Brus, F, 886

Bucci, TJ, 140

Buch, S, 506

Bucher, H-U, 332

Buchli, R, 113

Bucknall, MP, 324

Bührer, C, 336

Bui, KC, 878

Burdach, S, 716

Burke, JF, 17

Butcher, EC, 336

Cai, Z, 107

Cao, Y, 962

Cara, A, 792

Carling, DE, 1003

Carlton, DP, 30

Carreño, V, 618

Carver, TD, 754

Casado, J, 81

Castillo, I, 618

Castillo, L, 17

Cataltepe, O, 251

Celsi, G, 164

Chapman, TE, 17

Charles, R, 1018

Cheeseman, P, 568

Chen, D, 95

Chen, W, 173

Chen, Y, 292

Cheng, P-W, 42

Chern, J, 697

Chuai, J, 912

Churn, SB, 949

Ciccimarra, F, 564

Cirillo, L, 792

Ciurlionis, R, 638

Clark, DA, 768

Clark, JG, 851

Cochrane, CG, 211

Cockburn, F, 361

Cohen, F, 1009

Cole, DJ, 652

Colley, J, 1026

Corbett, RJT, 919

Cornaglia-Ferraris, P, 792

Cortizas, JA, 149

Côté, A, 926

Cranage, S, 533
Cromme-Dijkhuis, AH, 36

Cummings, JJ, 30

Cusack, NA, 993

Dacar, D, 198

Dalu, A, 140

Dancis, $J, 1$

Davidson, D, 11

Davis, JR, 471

Davis, LE, 722

Dawani, NH, 319

de Bruin, NC, 411

de Dávila, MTG, 592

Degenhart, HJ, 411

De Groot, A, 86

de Groot, R, 808

de Haan, AFJ, 124

Dekowsky, SA, 513

del Moral, T, 704

DeLorenzo, RJ, 949

DeMaria, A, 792

de Munick Keizer-Schrama, SMPF, 267

Demuth, SG, 543

den Hartog, H, 267

Denny, FW, Jr., 1036

de Ridder, MAJ, 267, 404

DeRojas-Walker, T, 17

de Vaan, GAM, 802

de Wolf, BTHM, 528

Dhawan, A, 568

Dillion, SB, 543

Dilloo, D, 716

Dosescu, J, 574

Dressel, MVC, 280

Drop, SLS, 267

Duc, G, 332

Du Caju, MVL, 607

Dudenhausen, JW, 336

Dzimiri, N, 55

Eblé, A, 962

Egeler, RM, 86, 471

Eichler, HG, 998

Elitsur, Y, 574

El-Mir, MY, 156

Eloby-Childress, S, 768

Elzenga, NJ, 886

Eriksson, UJ, 598

Esler, AL, 754

Esterman, AL, 1

Evin, MG, 709

Fabbri, R, 974

Facchinetti, F, 91

Facklam, RR, 988

Fadley, F, 55

Farquharson, J, 361

Felipe, A, 81

Fennessey, PV, 775

Ferriero, DM, 912

Festen, C, 124
Fike, CD, 30

Finkelstein, JN, 49

Fitzgibbons, JC, 306

Flamigni, C, 974

Flego, L, 397

Fleischman, SJ, 539

Floros, J, 668, 870

Fomon, SJ, 373

Fordyce, WE, 61

Förster, H-H, 812

Forster, J, 998

Forsythe, DW, 49

Foster, CM, 763

Frank, L, 292

Frantz, JA, 373

Fredholm, BB, 312

Friehs, I, 198

Fuchs, J, 555

Fuller, CR, 173

Fusco, M, 397

Gahl, WA, 579

Galal, O, 55

Garcia, D, 919

García-Mayor, RV, 149

Garg, M, 878

Garrett, JS, 1003

Gehrmann, J, 113

Genazzani, AD, 91

Genazzani, AR, 91

Gernhold, U, 336

Geven, WB, 124

Ghaffarifar, S, 993

Ghibu, F, 506

Ghizzoni, L, 91

Gidday, JM, 306

Giovannini, M, 262

Girardi, NL, 539

Gittenberger-De Groot, AC, 342

Givner, LB, 551

Gleason, CA, 943

Goldberg, RN, 704

Gonzales, LW, 668

González-Lestón, D, 149

Graulich, J, 336

Gray, L, 551

Green, M, 988

Gregory, ME, 61

Grieves, KC, 1003

Groener, JEM, 103

Gross, M, 543

Gross, TG, 471

Gunnar, MR, 1009

Guralnick, DE, 851

Habersang, K, 716

Hågå, P, 729

Hagberg, H, 312

Hăger, A, 955

Hählen, K, 86, 802

Halken, S, 182

Halley, DJJ, 629 
Hallman, M, 676

Halvorsen, S, 729

Haque, AK, 857

Harding, R, 690

Harel, Z, 747

Hashimoto, N, 763

Hauk, P, 998

Haworth, SG, 25

Hay, WW, Jr., 754

Heikinheimo, M, 797

Heiskanen, K, 740

Heitjan, DF, 251

Hepburn, T, 543

Herschkowitz, N, 657

Hersey, K, 107

Hess, J, 36

Hicar, M, 638

Hindmarsh, PC, 962

Hohimer, R, 722

Hokken-Koelega, ACS, 86, 267

Holmberg, C, 366

Holzinger, A, 844

Homoki, J, 76

Hooijkaas, H, 404

Hooper, SB, 690

Hop, WCJ, 808

Høsti, A, 182

Howatson, AG, 361

Hull, D, 1026

Husby, S, 182

Hyman, PE, 579

Ichikawa, K, 187

Ikegami, M, 709

Illi, O, 113

Imanishi, J, 501

İnan, C, 685

Ingelfinger, J, 579

Itoh, M, 187

Iughetti, L, 91

Iwamoto, HS, 844

Iwamoto, LM, 280

Jamieson, C, 361

Jansson, L, 598

Jiang, JD, 390

Jobe, AH, 709

Johnston, MV, 644

Jokinen, E, 522

Jones, TW, 539

Josting, A, 716

Kadison, A, 932

Kadowitz, PJ, 905

Kalayci, O, 685

Kållberg, N, 7

Kamel, A, 418

Kamm, KE, 697

Kandil, HM, 173

Kapitulnik, J, 258

Karen, P, 356

Kari, MA, 676

Karlberg, J, 733

Kaufmehl, K, 998

Kawano, Y, 187

Keel, M, 332

Keenan, A, 49

Keeney, SE, 857

Keil, LC, 30

Keku, EO, 173

Kelly, CM, 471
Kelly, FJ, 286

Kheiter, A, 211

Kilian, M, 182

Kiliç, I, 685

Kilinç, K, 685

Kirby, ML, 485

Kisker, CT, 169

Kita, M, 501

Klaessens, JHGM, 124

Kleijer, WJ, 103

Koh, SJG, 574

Koistinen, R, 366

Kolář, F, 892

Kollée, LAA, 124

Komai, H, 25

Koopman-Esseboom, C, 404

Koppenhafer, SL, 221

Kotiloğlu, E, 685

Kou, K, 187

Kraujalis, MJ, 306

Kreis, R, 657

Krensky, AM, 275

Kretschmar, T, 812

Kroos, MA, 103

Kugelman, A, 878

Kuusela, P, 797

Kydon, DW, 228

Lahdenne, P, 797

Lamers, WH, 1018

Lanning, M, 797

Lanyi, A, 471

Lapinleimu, H, 522

Laptook, AR, 919

Larsson, L, 955

Leary, HL, Jr., 173

Lecce, JG, 173

Lee, JD, 1

Lee, W-S, 864

Leffler, CW, 119

Leino, A, 522

Lemmen, RJ, 267

Levy, M, 25

Lew, CD, 878

Liem, KD, 124

Liest $\varnothing 1$, K, 729

Liley, HG, 668

Liliemark, E, 7

Liliemark, J, 7

Lin, J, 704

Lindgren, A-C, 418

Ling, EWY, 981

Link, G, 585

Little, D, 324

Liu, H, 905

Liu, S-C, 173

Liu, Y, 501

Logan, RW, 361

Lopez-Salva, A, 156

Lubec, G, 286

Macardle, PJ, 397

Macias, RIR, 156

Macrelli, S, 974

Madtes, DK, 851

Magnusson, P, 955

Majumdar, APN, 574

Mak, RHK, 379

Makhoul, IR, 878

Manco-Johnson, M, 169

Marchione, K, 539
Marcus, C, 418

Margery, V, 418

Marin, JJG, 156

Marinkovich, GA, 280

Martin, E, 113

Martinez, O, 704

Mast, F, 342

Mathews, MJ, 857

Mathews, MS, 896

Matsuda, Y, 722

Mawdsley, C, 506

McDonough, KH, 228

Meehan, B, 926

Mehendale, HM, 140

Meijer, AJ, 1018

Merritt, TA, 211

Miller, CE, 783

Miller, MJS, 768, 905

Mills, EL, 981

Mirza, M, 568

Mischke, D, 812

Mitsui, Y, 501

Mohan, P, 579

Mohapatra, NK, 42

Moliken, W, 237

Monte, MJ, 156

Moore, A, 506

Moore, DK, 543

Moorji, A, 55

Moser, T, 332

Moshier, JA, 574

Mowat, AP, 568

Mukouyama, T, 187

Mulder, PGH, 342

Mullis, PE, 962

Nakajima, T, 187

Nakamura, KT, 280

Nardo, L, 690

Narkewicz, MR, 775

Narus, JC, 1003

Nathanielsz, PW, 528

Navas, S, 618

Neijens, HJ, 808

Nelson, SE, 373

Noma, T, 187

Norstedt, G, 418

Nowicki, PT, 783

Noya, FJD, 981

Nuyt, AM, 896

Obladen, M, 336

Oeseburg, B, 124

Okano, M, 471

Okken, A, 886

Olivié, MAA, 149

Onouchi, Z, 501

Oostra, BA, 629

Op De Beeck, L, 607

Osborne, S, 652

Osborne, TN, 652

O'Shea, TM, 551

Oštádal, B, 892

Oštádalová, I, 892

Otto, J, 998

Oyaizu, N, 384

Øyasaeter, S, 205

Ozand, PT, 324

Pácha, J, 356

Padbury, JF, 709
Page, WV, 896

Pahwa, S, 384

Palumbo, A, 564

Paradisi, R, 974

Paradiso, AM, 42

Park, TS, 306

Parker, JC, 42

Pastor-Anglada, M, 81

Patrick, WJA, 361

Pearce, WJ, 493, 652

Peart, I, 319

Penzien, J, 657

Perheentupa, J, 740

Perlmutter, DH, 830

Peters, V, 390

Peterson, C, 7

Pexieder, T, 892

Pichoff, BE, 280

Pierce, MR, 768, 905

Pierce, RL, 905

Pietsch, T, 555

Pietz, J, 657

Pirruccello, SJ, 471

Pitkänen, S, 797

Pluchinotta, V, 974

Pohlová, I, 356

Polk, DH, 709

Porcu, E, 974

Porras, H, 926

Porter, TG, 543

Post, M, 506, 864

Poulain, FR, 30

Qin, F, 485

Rajantie, J, 797

Randolph, JF, Jr., 763

Rashed, MS, 324

Rating, D, 657

Ratjen, F, 664

Rayani, HH, 870

Refsum, HE, 729

Reichelt, KL, 205

Reuser, AJJ, 103

Rhoads, JM, 173

Rhodes, PG, 107

Ricard, G, 981

Rindler, MJ, 1

Risteli, J, 568

Risteli, L, 568

Riva, E, 262

Rivarola, MA, 592

Roberton, D, 397

Robillard, JE, 896

Roboz, JP, 390

Rödl, S, 198

Rodriguez-Bravo, T, 156

Rognum, TO, 205

Rönnemaa, T, 522

Rooman, RP, 607

Rose, SR, 967

Rosenfeld, CR, 697

Ross-Ascuitto, NT, 228

Routi, T, 522

Rudolph, AM, 243, 938

Ruggeri, S, 974

Ruiz-Moreno, M, 618

Rumpel, H, 113

Russo, P, 237

Ryan, RM, 49 
Sakata, K, 501

Salmenperä, L, 740

Salo, P, 522

Sanchez, M, 17

Sanchez, V, 618

Santoro, P, 564

Sarna, S, 366

Sarno, T, 564

Sas, TCJ, 404

Sauer, PJJ, 404

Saugstad, OD, 205

Schasfoort, M, 886

Scheidt, A, 716

Schlesinger, M, 390

Schmalstieg, FC, 857

Schmidt, D, 555

Schmitt, M, 812

Schoemaker, RC, 808

Scholz, TD, 221

Schutte, BC, 896

Schwab, KO, 998

Schwartz, AL, 835

Schwartz, B, 988

Scriver, CR, 819

Sedmak, DD, 1003

Seemayer, TA, 471

Segar, JL, 896

Segura-Dominguez, A, 149

Seidenberg, J, 218

Serfass, RE, 373

Serrano, MA, 156

Setty, BNY, 95

Shaddy, RE, 1003

Shah, AR, 306

Shah, R, 988

Shannon, D, 17

Shapiro, SM, 949

Shaywitz, BA, 539

Shaywitz, SE, 539

Sheldon, RA, 912

Shibata, M, 119

Shiota, T, 722

Shuman, H, 668

Sigrest, T, 107

Siimes, MA, 740, 797

Silberbach, M, 722

Silliman, CC, 993

Silver, S, 258

Silverman, CL, 543

Simell, O, 522
Sipalä, I, 366

Sjöström, B, 7

Smits, TM, 528

Snyder, JM, 513

Sohmer, H, 258

Song, L, 506

Sosenko, IRS, 292

Sousa, TR, 149

Spangenberg, P, 664

Stammers, J, 1026

Stephenson, T, 1026

Sterett, R, 919

Stibenz, D, 336

Stoltenberg, L, 205

Stonestreet, BS, 67

Storm, H, 205

Strandjord, TP, 851

Stuart, MJ, 95

Stuart-Smith, K, 25

Suguihara, C, 704

Sumegi, J, 471

Swanson, NJ, 993

Sweezey, N, 506

Tamborlane, WV, 539

Tan, ND, 11

Tangerman, A, 579

Tannenbaum, GS, 747

Tannenbaum, S, 17

Taylor, G, 543

Taylor, GA, 644

Teitel, DF, 243, 938

Teller, WM, 76

Terndrup, TE, 61, 932

Thaler, MM, 349

Thompson, RC, 704

Thorén, M, 418

Thureen, PJ, 775

Tjoa, S, 775

Tollefsbol, G, 919

Torres, A, 479

Towfighi, J, 251

Trapnell, BC, 844

Traystman, RJ, 644, 943

Trescher, WH, 644

Trivedi, P, 568

Trojan, S, 262

Tromp, CG, 86

Trost, A, 664

Tschann, JM, 1009
Tucker, DC, 479

Tulloh, RMR, 25

Tureaud, J, 574

Uchańska-Ziegler, B, 812

Uiterwijk, M, 194

Ulshen, MH, 173

Urbanek, R, 998

Vajro, P, 349

Välimäki, I, 522

Vallette, JD, Jr., 704

Van Acker, KJ, 886

van den Anker, JN, 808

Van der Auwera, JC, 886

van der Does-van den Berg, A, 802

van der Ende, J, 612

van der Heijden, BJ, 808

van der Kraan, M, 103

van der Ploeg, AT, 103

van der Zwan, CW, 404

van Diggelen, OP, 103

Van Dongen-Melman, JEWM, 86

Vannucci, RC, 251

Van Overmeire, B, 886

van Vliet, AM, 36

van Weel-Sipman, M, 802

van Weerden, JF, 802

Van Wyk, JJ, 1032

Veerman, AJP, 802

Venturoli, S, 974

Verhulst, FC, 612

Viggiano, D, 564

Vihervuori, E, 366

Viikari, J, 522

Visser, HKA, 411

Voelker, CA, 768

Vöhringer, R, 716

von der Hardt, H, 218

von Schweinitz, D, 555

von Siebenthal, K, 332

Wachter, UA, 76

Waddell, AE, 228

Wagener, WC, 988

Wagerle, LC, 237

Wagner, CL, 49

Wagner, JK, 962

Wahn, H-U, 812
Wald, ER, 988

Waldman, WJ, 1003

Wang, X-P, 384

Wang, Z-M, 164

Wara, D, 1009

Warbritton, A, 140

Warshaw, JB, 870

Wäsch, R, 812

Weaver, TE, 844

Weindling, AM, 319

Weisglas-Kuperus, N, 404

Welker, J, 664

Welte, K, 555

Wentzel, P, 598

Westerterp, KR, 411

Whitsett, JA, 844

Widness, JA, 67

Wiesemann, H-G, 664

Wilkinson, MH, 533

Wladimiroff, JW, 342

Wolf, M, 332

Woo, P, 932

Woodard, JP, 173

Woods, LL, 722

Woudstra, BR, 528

Wudy, SA, 76

Wulbrand, H, 298

Wyde, PR, 543

Yakubu, MA, 119

Yankaskas, JR, 42

Yasui, M, 164

Yata, J, 187

Yoshizawa, I, 187

Young, VR, 17

Yoxall, CW, 319

Yu, YM, 17

Yue, N, 668

Zempleni, J, 585

Zezschwitz, G, 298

Zhang, X-J, 768

Ziegler, A, 812

Ziegler, EE, 373

Zijlstra, WG, 528

Zimmermann, LJI, 864

Zobel, G, 198

Zola, H, 397

Zurcher, SD, 493 